\title{
Jumping mode scanning force microscopy: a suitable technique for imaging DNA in liquids
}

\author{
F. Moreno-Herrero*, P.J. de Pablo, M. Álvarez, J. Colchero, \\ J. Gómez-Herrero, A.M. Baró \\ Laboratorio de Nuevas Microscopías, Departamento de Física de la Materia Condensada, \\ Universidad Autónoma de Madrid, E-28049 Madrid, Spain
}

\begin{abstract}
In this work, we introduce jumping mode (JM) scanning force microscopy (SFM) as a suitable technique for imaging soft samples in liquid environment like DNA adsorbed on mica. JM reveals as a non-intrusive technique where shear forces are minimized by performing the scanning motion without tip-sample contact. We find no visible damage on DNA samples and the nominal height of $2 \mathrm{~nm}$ of the molecules is achieved when imaging applying a maximum normal force of $\sim 150 \mathrm{pN}$. In addition to topographic images, adhesion maps of DNA are simultaneously recorded showing that the minimum adhesion force occurs on top of the DNA molecules.
\end{abstract}

(C) 2003 Elsevier Science B.V. All rights reserved.

Keywords: Scanning force microscopy; Atomic force microscopy; DNA; Jumping mode

In the last 50 years the advances in molecular biology have been spectacular. Physicists have contributed to this enormous growth with the development of powerful techniques such as nuclear magnetic resonance (NMR), electron microscopy or X-ray crystallography. Although the resolution of these techniques is outstanding, they still have important disadvantages. In many cases, samples have to be in vacuum conditions or prepared as crystals. Also, these techniques are not single molecule techniques thus all the molecules are treated as they worked in the same way and as its mean behavior would control its biological function.

Scanning force microscopy (SFM) [1], also called atomic force microscopy, is a single molecule technique which allows imaging the surface of a wide variety of samples in many different environments.

\footnotetext{
${ }^{*}$ Corresponding author.

E-mail address: fernando.moreno@uam.es (F. Moreno-Herrero).
}

During the last 15 years, SFM has been used to study solid-vacuum [2,3], solid-gas (typically ambient air) [1] and solid-liquid interfaces [4], specially soft samples like DNA [5]. In this third field SFM appears as a unique technique to resolve biological structures at the molecular level under physiological conditions.

In this work we introduce jumping mode (JM) [6] also called pulsed force microscopy by other authors [7] as a suitable technique for imaging soft samples like DNA under liquid environment. Both modes essentially work in a similar manner but while pulsed force microscopy requires additional hardware with respect to classic contact mode, JM implementation is just a software routine running in a digital signal processor memory, therefore is more flexible than pulsed force microscopy. Our results show that JM is a non-intrusive technique thus no visible damage is induced in the samples during scanning. Using JM with very soft cantilevers (force constant: $20 \mathrm{mN} / \mathrm{m}$ ) 
in liquid, DNA images show a molecular height of $2 \mathrm{~nm}$ in good agreement with other techniques [8]. This result is particularly relevant because experiments performed using DSFM show DNA images with a height of less than half of the nominal height of the DNA [9-18]. The anomalous height is explained in terms of dehydration of the molecules [10]; presence of salt layers [19]; tip compression [11] or strong interaction between DNA and substrate which compress the molecules to the surface [20]. In addition to the acquisition of topographic images, JM allows to obtain adhesion maps of samples [21]. In this work we also present adhesion images of the adsorbed DNA molecules. Our results show that DNA presents a negligible adhesion to the tip. We have found that this result can be extended to other samples such as the bacteriophage $\phi 29$ [22,23] or the Paired Helical Filaments of the Alzheimer Disease [24] (data not shown).

Imaging soft samples in liquids is typically performed using DSFM. Due to the small Q-factor in liquids the sensitivity of DSFM is dramatically reduced respect to ambient air. The weakness of van der Waals forces in liquids (typically 10 times smaller that in air) and the long range character of the forces induced by the Debye double layer, i.e., the electrostatic interaction of the ions in the liquid medium $[25,26]$, makes non-contact operation in liquids extremely difficult. Therefore DSFM, as well as JM, operates in contact. However, while the force applied in DSFM is not directly known since the feedback is performed on the amplitude, in JM the maximum force is clearly determined by the feedback set-point as in classic contact mode.

In addition to drawbacks of basic nature, we believe that JM has clear advantages over DSFM also due to practical considerations. In this context, the first important issue is the ease of implementation. JM is just a software routine requiring no additional hardware with respect to classic contact mode and no special treatment of cantilever as for magnetic DSFM. In addition we recall that DSFM in liquids is much more difficult to operate as compared to its operation in air. First of all, cantilever oscillation driven by a small piezoelectric attached to the cantilever chip produces a frequency spectrum with many spurious high amplitude resonances due to the excitation of the liquid cell. This problem can be avoided by using a magnetic field to drive the cantilever [32], but then cantilevers have to be coated with a magnetic material that may lead to contamination of the samples. In addition, if the cantilever is very stiff the force applied by the magnetic field is too weak to induce sufficient motion of the tip and the method cannot be used. Magnetically covered cantilevers are expensive and difficult to purchase. Another important advantage of JM as compared to DSFM is the synchronization of the lateral and vertical movements, i.e., in JM the lateral displacement is always performed at the maximum tip-sample separation avoiding lateral forces induced by the scanning process. On the contrary, in DSFM in liquids is not clear whether or not the lateral motion is performed out of contact. Finally, JM offers the possibility to measure adhesion force maps. Adhesion is a fundamental magnitude in any realistic contact model. In contrast, DSFM offers phase maps that are extremely difficult to understand in terms of more basic magnitudes.

The absence of capillary forces allows to minimize the adhesion force and hence JM image acquisition with very low forces is possible by using very soft cantilevers. In Fig. 1 two force versus distance plots are shown. ${ }^{1}$ Fig. $1 \mathrm{~A}$ is performed in ambient air with a cantilever of $0.75 \mathrm{~N} / \mathrm{m}$ [28]. As can be seen adhesion forces of tens of $\mathrm{nN}$ are present due to capillary forces and a strong van der Waals interaction. As a consequence, when working in ambient conditions irreversible damage is present in soft samples when using JM [29]. The situation is completely different when imaging in liquids. Fig. 1B shows the force versus distance plot performed in liquid using a cantilever of $20 \mathrm{mN} / \mathrm{m}$ [30]. The weak van der Waals interaction as well as the absence of capillary forces minimizes adhesion forces allowing to obtain images with a maximum force of tens of pN. Fig. 1 shows that in liquids the forces can be reduced in more than two orders of magnitude.

In liquids, the interaction between molecules and substrate is weaker than in air and hence, methods for fixing the molecules to the surface are in continuous development. Ideally, these methods must firmly attach the molecules to the surface but without any conformational deformation which could inhibit its biological function. We have used 3-aminopropyltriethoxysilane (APTES) (Sigma A-3648) to attach the molecules to a

\footnotetext{
${ }^{1}$ Data has been collected using a commercial microscope from Nanotec Electronica TM, C/Padilla 1, Madrid, Spain [27].
} 

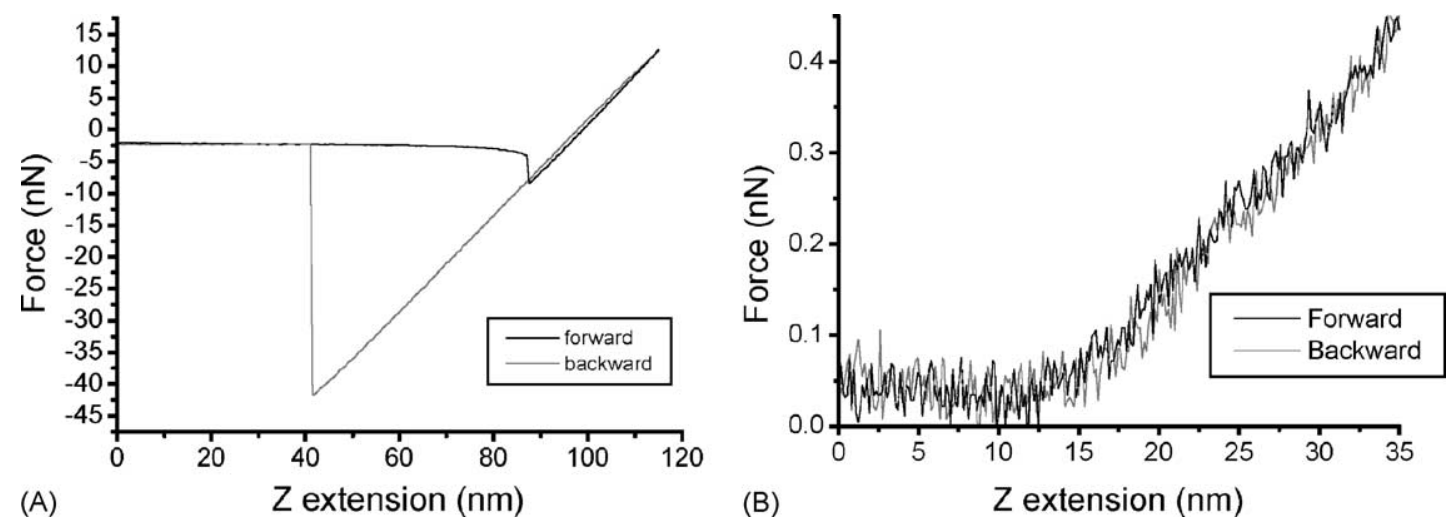

Fig. 1. Force versus distance plots performed in ambient air: (A) with a cantilever of $0.75 \mathrm{~N} / \mathrm{m}$ and under liquid; (B) with a cantilever of $20 \mathrm{mN} / \mathrm{m}$. Since in ambient air the high adhesion forces introduce irreversible damage on soft samples, in liquid the weak van der Waals interactions allows imaging applying extremely low normal forces.

mica substrate. A freshly cleaved mica sheet was immersed for $15 \mathrm{~min}$ in a $0.1 \%$ dilution of APTES in water and then rinsed with 2-propanol, water and dried in a gentle stream of nitrogen. Then, a drop of the DNA solution is placed on the treated mica for $10 \mathrm{~min}$ and afterwards rinsed with the appropriate buffer. The sample is placed in the liquid cell and never allowed to dry. The liquid cell is filled with $1 \mathrm{ml}$ of $20 \mathrm{mM}$ Tris ( $\mathrm{pH}$ 7.5), $10 \mathrm{mM} \mathrm{MgCl}$. This sample preparation protocol can be used as a general protocol for SFM imaging of biological samples in liquids.

As a test of JM we have used DNA adsorbed on mica. The low height of the DNA measured by SFM in ambient air has been a recurrent problem in the scientific community for the last few years. On the other hand, SFM is characterized by the high accuracy in the $Z$ dimension. To explain this apparent paradox, arguments like dehydration, presence of a salt layer, tip compression or strong molecule-surface interaction have been put forward. Imaging in liquids should solve the height problem of the DNA since any salt layer should be dissolved, the interaction moleculesubstrate minimized and the molecule fully hydrated. Fig. 2A shows the topographic image of DNA obtained with JM applying a maximum force of $140 \mathrm{pN}$. The quality and resolution of this image is comparable to that obtained in air using dynamic scanning force microscopy (DSFM) [31]. In Fig. 2B a topographic profile is shown. Data show that the height of the molecules is, within the experimental error, in agreement with the nominal height of the
DNA which is $2 \mathrm{~nm}$. Moreover, our data on DNA measured using magnetic DSFM [32] in liquids reveals a mean height of the molecules of $0.8 \mathrm{~nm}$ less than half of the value obtained in JM. This suggests that the force applied by the tip in DSFM is bigger than the $\sim 150 \mathrm{pN}$ applied with JM. Note that the width of the molecule is $\sim 10 \mathrm{~nm}$, showing a lateral resolution comparable or even better than DSFM in air probably due to the presence of contact of the tip with the molecule. Fig. $2 \mathrm{C}$ shows an adhesion map simultaneously obtained with the topography. The data show that the minimum adhesion occurs on the DNA molecule. An adhesion force profile is plotted in Fig. 2D. Adhesion forces usually never exceed $0.5 \mathrm{nN}$. This kind of experiments could be of great importance when using functionalized cantilevers to study specific interactions.

We have shown that JM is an appropriate technique for imaging soft samples in liquids. We have used DNA as a test sample obtaining reproducible images with no visible damage. The weak interactions present in liquid allows imaging applying extremely low forces. The measurement of the nominal height of DNA in liquids suggest that the height of the molecules is not affected by tip compression when imaging applying a maximum force of $\sim 150 \mathrm{pN}$ and that the chemical treatment of the surface is an appropriate sample preparation method for SFM imaging of biomolecules in liquid environment. In addition to topographic data, adhesion maps can be obtained with JM. Data show that the places where the adhesion is lower 
(A)

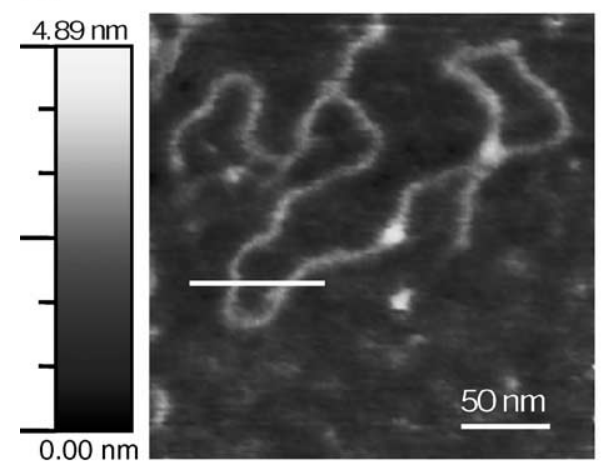

(C)

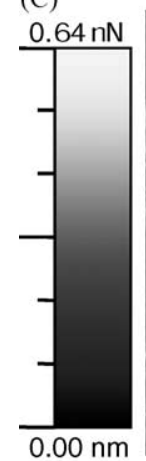

(B)
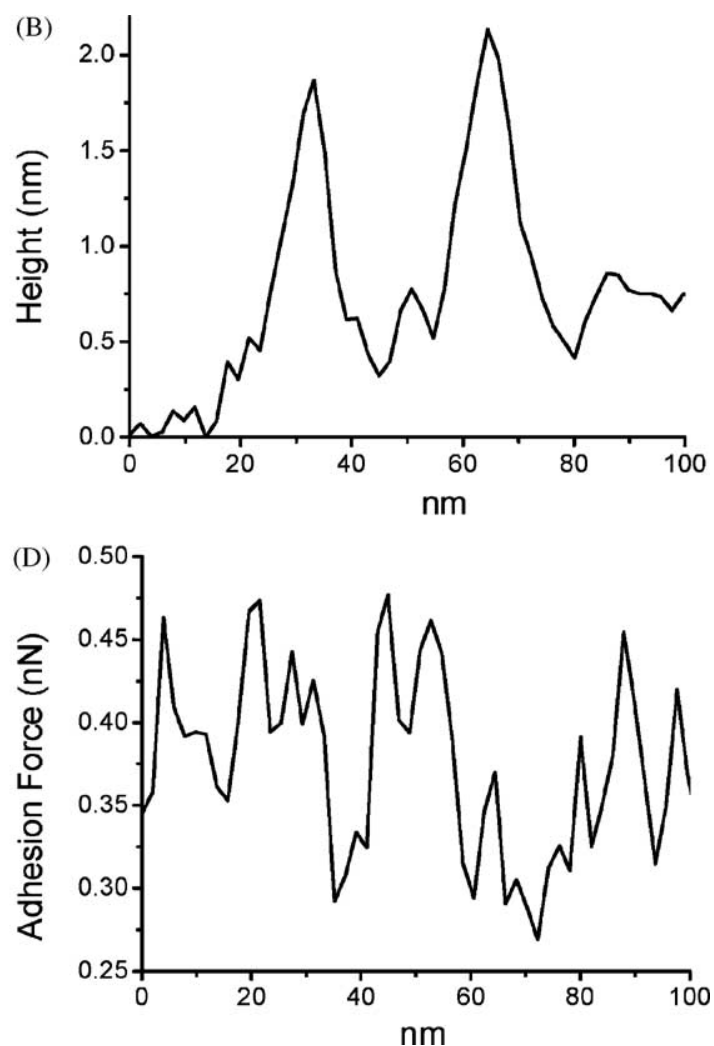

Fig. 2. Topographic (A) and adhesion (C) images of DNA adsorbed on mica obtained with JM. The quality and the resolution is comparable with the one exposed when imaged with DSFM. The topographic and adhesion profiles are, respectively, shown in parts (B) and (D).

is on top of the molecules suggesting that the chemical coverage of the surfaces introduces the adhesion measured out of the molecules.

\section{Acknowledgements}

We acknowledge support from the Comunidad de Madrid through a Ph.D. fellowship for F. MorenoHerrero. This work is supported by the Ministerio de Educación y Cultura through a DGESIC project no. BFM2001-0150 and MAT2001-0664.

\section{References}

[1] G. Binnig, C.F. Quate, C. Gerber, Phys. Rev. Lett. 56 (1986) 930-933.

[2] Y. Sugawara, M. Ohta, H. Veyana, S. Morita, Science 210 (1995) 1646.
[3] F.J. Giessibl, Science 267 (1995) 68.

[4] O. Marti, B. Drake, P.K. Hansma, Appl. Phys. Lett. 51 (1987) 484.

[5] H.G. Hansma, Annu. Rev. Phys. Chem. 52 (2001) 71-92.

[6] P.J. de Pablo, J. Colchero, J. Gómez-Herrero, A.M. Baro, Appl. Phys. Lett. 73 (1998) 3300-3302.

[7] A. Rosa-Zeise, E. Weilandt, S. Hild, O. Marti, Meas. Sci. Technol. 8 (1997) 1333.

[8] J.D. Watson, F.H.C. Crick, Nature 171 (1953) 737-738.

[9] H.G. Hansma, R.L. Sinsheimer, M.Q. Li, P.K. Hansma, Nucl. Acids Res. 20 (1992) 3585-3590.

[10] C. Bustamante, C. Rivetti, D.J. Keller, Curr. Opin. Struct. Biol. 7 (1997) 709-716.

[11] Y. Lyubchenko, L. Shlyakhtenko, R. Harrington, P. Oden, S. Lindsay, Proc. Natl. Acad. Sci. USA 90 (1993) 21372140.

[12] W.L. Shaiu, D.D. Larson, J. Vesenka, E. Henderson, Nucl. Acids Res. 21 (1993) 99-103.

[13] N.H. Thomson, S. Kasas, B. Smith, H.G. Hansma, P.K. Hansma, Langmuir 12 (1996) 5905-5908.

[14] H.G. Hansma, I. Revenko, K. Kim, D.E. Laney, Nucl. Acids Res. 24 (1996) 713-720. 
[15] Y.L. Lyubchenko, L.S. Shlyakhtenko, T. Aki, S. Adhya, Nucl. Acids Res. 25 (1997) 873-876.

[16] Y. Maeda, T. Matsumoto, T. Kaway, Appl. Surf. Sci. 140 (1999) 400-405.

[17] F. Moreno-Herrero, P. Herrero, J. Colchero, A.M. Baro, F. Moreno, FEBS Lett. 459 (1999) 427-432.

[18] J.Y. Ye, K. Umemura, M. Ishikawa, R. Kuroda, Anal. Biochem. 281 (2000) 21-25.

[19] J. Vesenka, S. Manne, G. Yang, C.J. Bustamante, E. Henderson, Scan. Microsc. 7 (1993) 781-788.

[20] F. Moreno-Herrero, J. Colchero, A.M. Baro, Ultramicroscopy, submitted for publication.

[21] P.J. de Pablo, J. Colchero, J. Gómez-Herrero, A.M. Baro, D.M. Schaefer, S. Howell, B. Walsh, R. Reifenberger, J. Adhesion 71 (1999) 339-356.

[22] E. Vinuela, A. Camacho, F. Jimenez, J.L. Carrascosa, G. Ramirez, M. Salas, Philos. Trans. Roy. Soc. Lond. B 276 (1976) 29-35.
[23] J.L. Carrascosa, E. Vinuela, N. Garcia, A. Santisteban, J. Mol. Biol. 154 (1982) 311-324.

[24] M. Kidd, Nature 197 (1963) 192-193.

[25] H.J. Butt, Biophys. J. 60 (1991) 777-785.

[26] T.J. Senden, C.J. Drummond, P. Kekicheff, Langmuir 10 (1994) 358.

[27] http://www.nanotec.es.

[28] Olympus Optical Co. (Europa) GMBH, Wenderstrasse 14-18, 20097 Hamburg.

[29] F. Moreno-Herrero, P.J. de Pablo, J. Colchero, J. GómezHerrero, A.M. Baró, Surf. Sci. 453 (2000) 152-158.

[30] Nanosensors GmbH \& Co, KG IMO-Building, Im Amtmann 6, D-35578 Wetzlar-Blankenfeld Germany.

[31] F. Moreno-Herrero, P. Herrero, J. Colchero, A.M. Baro, F. Moreno, Biochem. Biophys. Res. Commun. 280 (2001) 151-157.

[32] W. Han, S.M. Lindsay, T. Jing, Appl. Phys. Lett. 69 (1996) 4111-4113. 